\title{
S-curve Engineering for ON-state Performance using Anti-ferroelectric/Ferroelectric Stack Negative-Capacitance FinFET
}

\author{
Shih-En Huang, Student Member, IEEE, Pin Su, Member, IEEE, and Chenming Hu, Life Fellow, IEEE
}

\begin{abstract}
This work investigates the S-curve engineering by exploiting the anti-ferroelectric (AFE)/ferroelectric (FE) stack negative-capacitance FinFET (NC-FinFET) to improve both the subthreshold swing and $\mathrm{ON}$-state current (ION). The capacitance matching and $\mathrm{ON}$-state performance are evaluated by using a short-channel AFE/FE stack NC-FinFET model. Our study indicates that the AFE/FE gate-stack can theoretically achieve surprising improvements to the OFF-state current (IOFF) and IoN relative to IRDS projections. There is significant long-term advantage to IC power consumption and speed if materials with certain AFE and FE characteristics can be developed and introduced into IC manufacturing.
\end{abstract}

Index

metal-ferroelectric-insulator-semiconductor negative-capacitance field-effect transistor (NCFET),

Terms-FinFET, (MFIS)-type anti-ferroelectric

\section{INTRODUCTION}

$\mathrm{N}$ EGATIVE -capacitance field-effect transistor (NCFET) [1]-[12], especially NC-FinFET [2]-[10] has garnered substantial interest as the next-generation logic transistor because it can provide steeper subthreshold slope (SS $<60$ $\mathrm{mV} /$ dec.) and much reduced short-channel effects [7]-[12]. However, the performance of the NCFET depends on the capacitance matching between the ferroelectric capacitance $\left(\mathrm{C}_{\mathrm{fe}}\right)$ and the internal capacitance $\left(\mathrm{C}_{\text {int }}\right)$, which is challenging because both are strongly bias dependent [6]. Fig. 1(a) illustrates that for an NCFET with a single-layer ferroelectric, it is very difficult to obtain good capacitance matching all the way from subthreshold to strong inversion by changing the ferroelectric properties such as the remnant polarization $\left(\mathrm{P}_{\mathrm{r}}\right)$. The ON-state performance of the NCFET is usually degraded due to the degraded internal-voltage amplification $\left(A_{V}\right)[6]$ in strong inversion. It is possible to obtain the anti-ferroelectric (AFE) phase of doped $\mathrm{HfO}_{2}$ in addition to the ferroelectric (FE) orthorhombic phase [13]-[17]. In this paper, with the aid of a

This work was supported in part by "Center for the Semiconductor Technology Research" from The Featured Areas Research Center Program within the framework of the Higher Education Sprout Project by the Ministry of Education (MOE), Taiwan, and in part by the Ministry of Science and Technology, Taiwan, under contracts 110-2634-F-009-027 and 110-2218-E-A49-014-MBK.

S.-E. Huang and P. Su are with the Department of Electronics Engineering \& Institute of Electronics, National Chiao Tung University, Hsinchu 30010, Taiwan (e-mail of corresponding author: pinsu@faculty.nctu.edu.tw).

C. Hu is with the Department of Electrical Engineering and Computer Science, University of California at Berkeley, Berkeley, CA 94720 USA, and also with the International College of Semiconductor Technology, National Chiao Tung University, Hsinchu 30010, Taiwan.
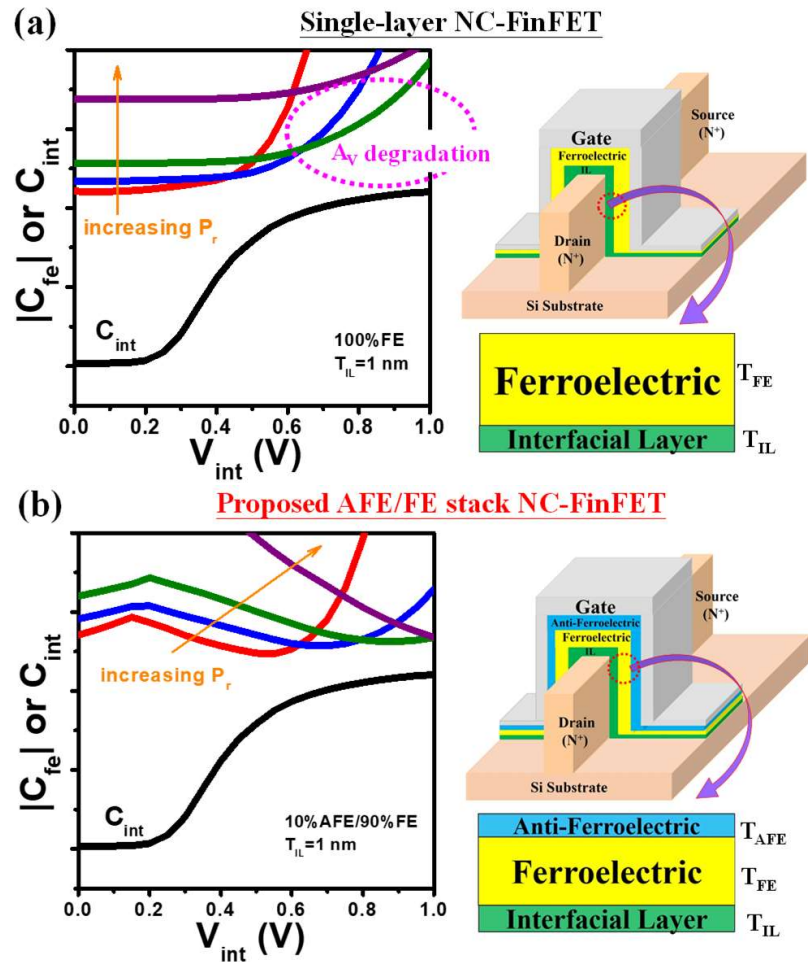

Figure 1. Capacitance matching at source end (i.e., $y=0$ ) with various $\mathrm{P}_{\mathrm{r}}$ and schematic for MFIS-type (a) single-layer and (b) AFE/FE stack NC-FinFET. Fig. 1(a) indicates that, albeit reducing $P_{r}$ improves capacitance matching in the subthreshold region, it degrades capacitance matching in strong inversion. The doping concentrations of source/drain and channel $\left(\mathrm{N}_{\mathrm{ch}}\right)$ are $1 \mathrm{E} 20 \mathrm{~cm}^{-3}$ and $1 \mathrm{E} 15$ $\mathrm{cm}^{-3}$, respectively. Abrupt junction is assumed. $\mathrm{T}_{\mathrm{AFE}}, \mathrm{T}_{\mathrm{FE}}$ and $\mathrm{T}_{\mathrm{IL}}$ are thicknesses of anti-ferroelectric, ferroelectric and interfacial-layer, respectively.

developed short-channel AFE/FE stack NC-FinFET model, we propose to boost the $\mathrm{ON}$-state performance by engineering the S-curve of the metal-ferroelectric-insulator-semiconductor (MFIS) type AFE/FE stack NC-FinFET. [Fig. 1(b)]

This paper is organized as follows. In Section II, we present our methodology for constructing the MFIS-type AFE/FE stack NC-FinFET model. In Section III, by using a short-channel AFE/FE stack NC-FinFET model calibrated with published FinFET data [19], the capacitance matching and ON-state performance of the AFE/FE stack NC-FinFET are evaluated. Based on IRDS [20] parameters, the projection of the performance of the AFE/FE stack NC-FinFET for future technology nodes will be investigated in section IV. Finally, Section V draws the conclusion. 


\section{Methodology}

In this section, a short-channel MFIS-type AFE/FE stack NC-FinFET model compatible with BSIM-CMG is developed. Without an internal metal between the ferroelectric and interfacial layer, Fig. 1(b) shows a schematic sketch of the MFIS-type AFE/FE stack NC-FinFET structure modeled in this work. Note that different from the MFMIS-type NC-FinFET with a uniform polarization charge distribution in the ferroelectric, the internal charge in the MFIS-type gate stack varies with the channel position y (which is known as the MFIS-specific distributed charge effect) [8][9]. Based on the surface potential-based scheme of BSIM-CMG, the internal charge, $\mathrm{Q}_{\text {int }}$, and internal voltage, $\mathrm{V}_{\text {int }}$, of the NC-FinFET can be determined as follows:

$$
\begin{aligned}
& Q_{\text {int }}(y)=C_{o x}\left(\begin{array}{c}
V_{\text {int }}(y)-V_{f b}-V-V_{t} \ln \left(\frac{8 \varepsilon_{s} V_{t} N_{c h}}{q n_{i}^{2} W_{\text {fin }}^{2}}\right) \\
-2 V_{t h} \ln \left(\frac{\beta}{\cos \beta}\right)-\varphi_{\text {per }}
\end{array}\right)
\end{aligned}
$$

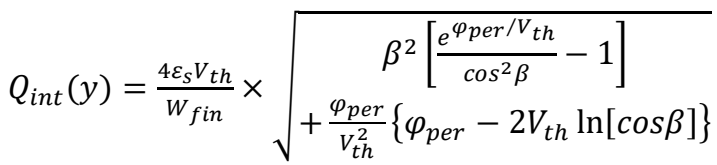

$$
\begin{aligned}
& V_{i n t}(y)=V_{g s}-V_{A F E}(y)-V_{F E}(y)
\end{aligned}
$$

where $\mathrm{V}_{\mathrm{fb}}$ is the flat-band voltage, $\varepsilon_{\mathrm{s}}$ the permittivity of the channel, $\mathrm{n}_{\mathrm{i}}$ the intrinsic carrier concentration, $\mathrm{V}_{\text {th }}$ the thermal-voltage given by $\mathrm{k}_{\mathrm{B}} \mathrm{T} / \mathrm{q}$ and $\mathrm{V}$ the quasi-Fermi potential. Note that the internal-voltage $\left(\mathrm{V}_{\mathrm{int}}\right)$ varies with the channel position, which is related to the voltage drop across the anti-ferroelectric $\left(\mathrm{V}_{\mathrm{AFE}}\right)$ and ferroelectric $\left(\mathrm{V}_{\mathrm{FE}}\right)$. We describe the anti-ferroelectric and ferroelectric properties by using the Kittel model [15][21] and Landau-Khalatnikov (L-K) equation [22], respectively:

$V_{A F E}(y)=T_{A F E}\left[\begin{array}{c}\left(2 \alpha_{p}+\alpha_{n}+12 \beta_{A F E} P_{a}^{2}\right) Q_{i n t}(y) \\ -P_{a}\left(\alpha_{n}+18 \beta_{A F E} Q_{i n t}^{2}(y)\right) \\ -\beta_{A F E}\left(2 P_{a}-Q_{i n t}(y)\right)^{3}+7 \beta_{A F E} Q_{i n t}^{3}(y)\end{array}\right]$

$V_{F E}(y)=T_{F E}\left[2 \alpha_{F E} Q_{i n t}(y)+4 \beta_{F E} Q_{i n t}^{3}(y)\right]$

where $\alpha_{\mathrm{p}}, \alpha_{\mathrm{n}}, \beta_{\mathrm{AFE}}$ and $\mathrm{P}_{\mathrm{a}}$ are anti-ferroelectric parameters, while $\alpha_{\mathrm{FE}}$ and $\beta_{\mathrm{FE}}$ are ferroelectric parameters.

The Kittel model [Eqn. (3-b)] and L-K equation [Eqn. (3-c)] have been seamlessly integrated into a unified surface potential equation. By self-consistently solving Eqn. (1), (2) and (3) under a given bias condition, the internal charge, $Q_{\text {int }}$, can be iteratively determined.

We have verified our Q-V model using TCAD numerical simulation [24]. Fig. 2(a) and (b) show the $Q_{\text {int }}$ and corresponding $\mathrm{V}_{\text {int }}$ at the source end. It can be seen that the $\mathrm{Q}_{\text {int }}$ [Fig. 2(a)] for the AFE/FE stack case is larger than that of the single-layer one. Due to the negative capacitance effect, the internal voltage change can be greater than the change in applied voltage. With the impact of AFE, the $\Delta \mathrm{V}_{\text {int, } \mathrm{AFE} / \mathrm{FE}}$ is larger than the $\Delta \mathrm{V}_{\mathrm{int}, \mathrm{FE}}$ for a given gate-bias change $\left(\Delta \mathrm{V}_{\mathrm{GS}}\right)$ [as
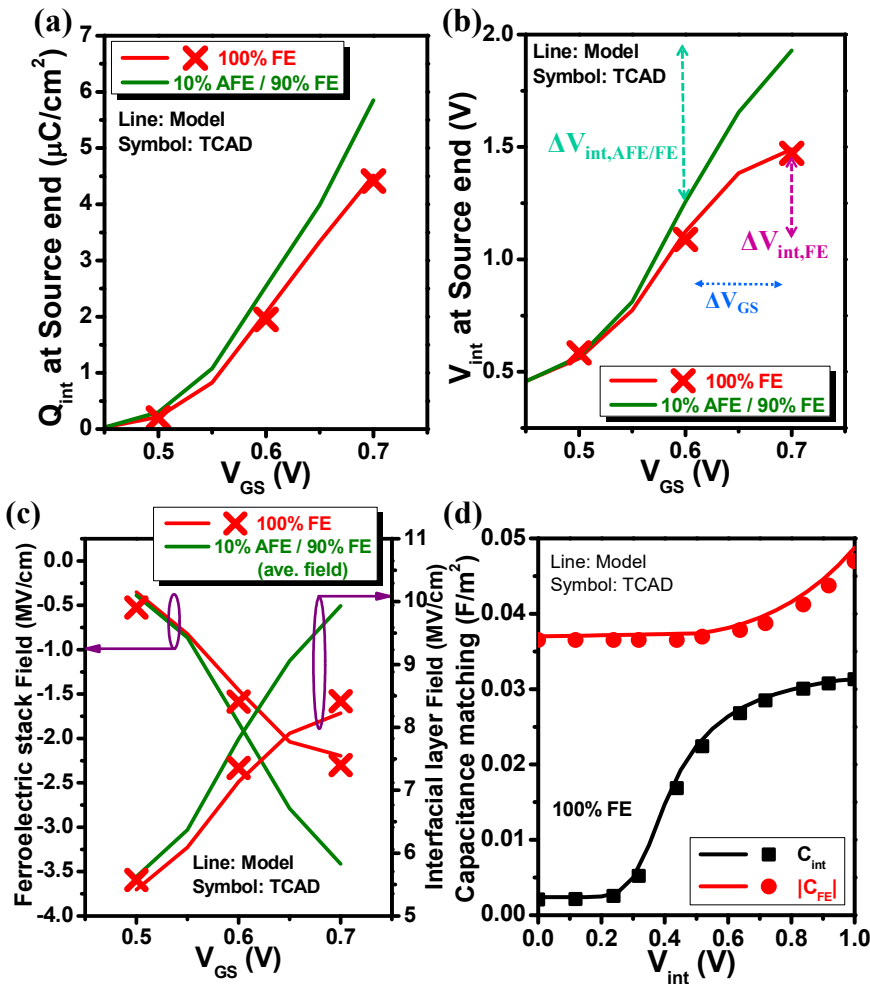

Figure 2. Calculated (a) internal charge, $Q_{\text {int }}$, and (b) internal voltage, $V_{\text {int }}$, at the source end with varying $\mathrm{V}_{\mathrm{GS}} \Delta \mathrm{V}_{\mathrm{int}, \mathrm{AFE} / \mathrm{FE}}$ and $\Delta \mathrm{V}_{\mathrm{int}, \mathrm{FE}}$ in (b) are the changes in $\mathrm{V}_{\text {int }}$ for the AFE/FE stack and the single-layer FE, respectively, due to the change in $\mathrm{V}_{\mathrm{GS}}$. (c) Vertical electric field in the ferroelectric stack and interfacial layer at the source end. (d) Verification of capacitance matching at the source end for the single-layer FE case. The thicknesses of ferroelectric stack and interfacial-layer are $3.6 \mathrm{~nm}$ and $1 \mathrm{~nm}$, respectively.

shown in Fig. 2(b)]. In addition, Fig. 2(c) further shows the vertical field in the ferroelectric stack and interfacial layer at the source end. It can be seen that the vertical field in the ferroelectric stack is opposite to that in the interfacial layer, and the average vertical field of the AFE/FE stack is larger than the single-layer FE one. Fig. 2(d) shows the comparison of our model-calculated capacitance matching with TCAD simulation [24] for the single-layer FE case.

Using the surface potential-based scheme, the drain current can be derived [8]:

$I_{d s}=\frac{2 \mu_{n} H_{f i n}}{L_{g}}\left[F\left(Q_{i n t, d}\right)-F\left(Q_{i n t, s}\right)\right]$

where

$F\left(Q_{\text {int }}\right)=A_{4} Q_{\text {int }}^{4}+A_{3} Q_{\text {int }}^{3}+A_{2} Q_{\text {int }}^{2}+A_{1} Q_{\text {int }}$

$A_{1}=V_{t h}$

$$
\begin{aligned}
& A_{2}=-T_{A F E}\left[\begin{array}{c}
\frac{1}{2}\left(2 \alpha_{p}+\alpha_{n}+24 \beta_{A F E} P_{a}^{2}\right) \\
+12 \beta_{A F E} P_{a} q N_{c h} W_{f i n}-3 \beta_{A F E} q^{2} N_{c h}^{2} W_{\text {fin }}^{2}
\end{array}\right] \\
& -T_{F E}\left[\alpha_{F E}+\frac{3}{2} \beta_{F E} q^{2} N_{c h}^{2} W_{f i n}^{2}+\frac{1}{2 C_{o x} T_{F E}}\right] \\
& A_{3}=T_{A F E}\left(16 \beta_{A F E} P_{a}-8 \beta_{A F E} q N_{c h} W_{f i n}\right) \\
& -4 T_{F E} \beta_{F E} q N_{c h} W_{\text {fin }} \\
& A_{4}=-6 T_{A F E} \beta_{A F E}-3 T_{F E} \beta_{F E}
\end{aligned}
$$




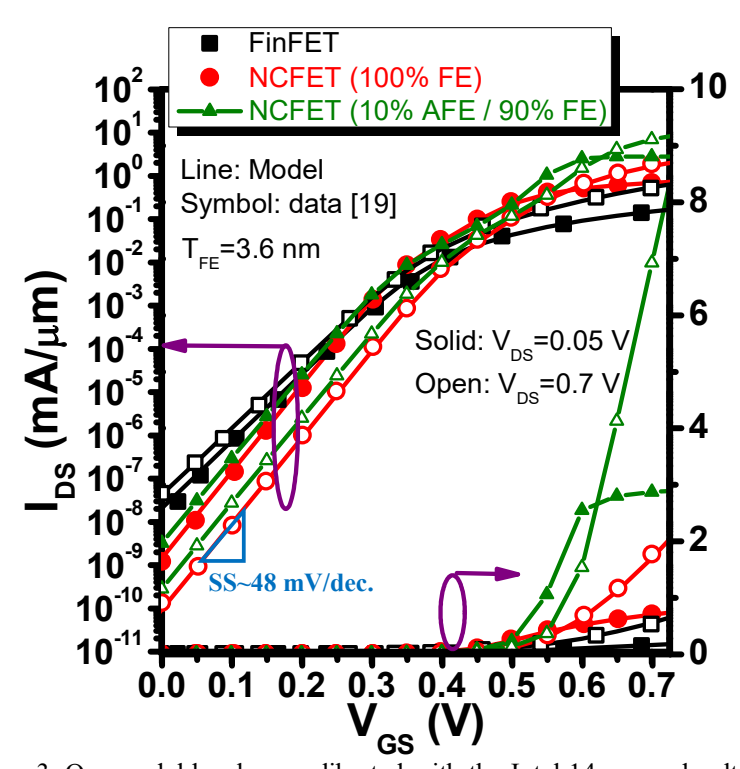

Figure 3. Our model has been calibrated with the Intel 14-nm node ultra-low power (ULP) FinFET technology [19]. Intrinsic $\mathrm{I}_{\mathrm{ON}}$ can be further improved for the AFE/FE stack NC-FinFET.
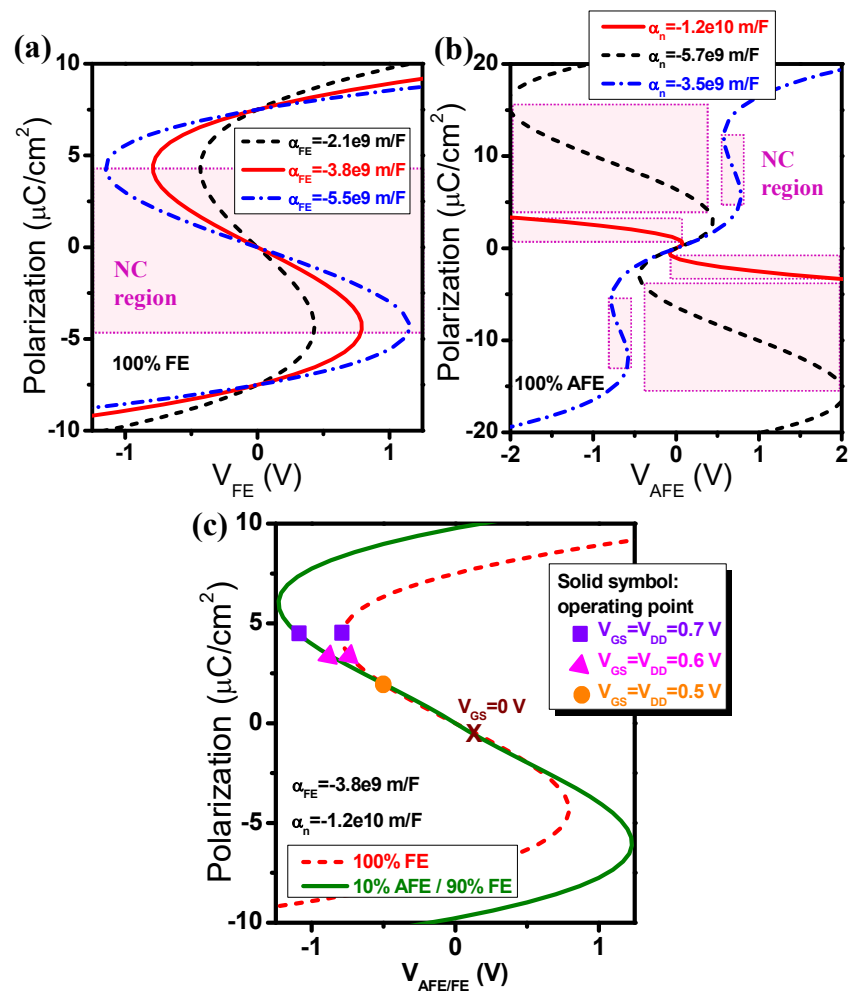

Figure 4. P-V curves for (a) $100 \% \mathrm{FE}$ with various $\alpha_{\mathrm{FE}}$, (b) $100 \% \mathrm{AFE}$ with various $\alpha_{\mathrm{n}}$, and (c) the AFE/FE stack. The symbols in (c) shows the operating points at different $\mathrm{V}_{\mathrm{GS}}$, indicating that the AFE/FE stack can extend the negative capacitance region and improve the $A_{V}$ in strong inversion.

In addition, the $\mathrm{V}_{\mathrm{DS}}$ and gate-length dependences in SS and $\mathrm{V}_{\mathrm{T}}$ [10][11] are physically modeled with the analytical short-channel Qint model [8].

The model is calibrated against the data of the Intel 14-nm node ultra-low-power (ULP) FinFET [19]. Fig. 3 shows a good agreement with the experiment data of FinFET. It also shows the hysteresis-free $\mathrm{I}_{\mathrm{DS}}-\mathrm{V}_{\mathrm{GS}}$ for both AFE/FE stack and (a)
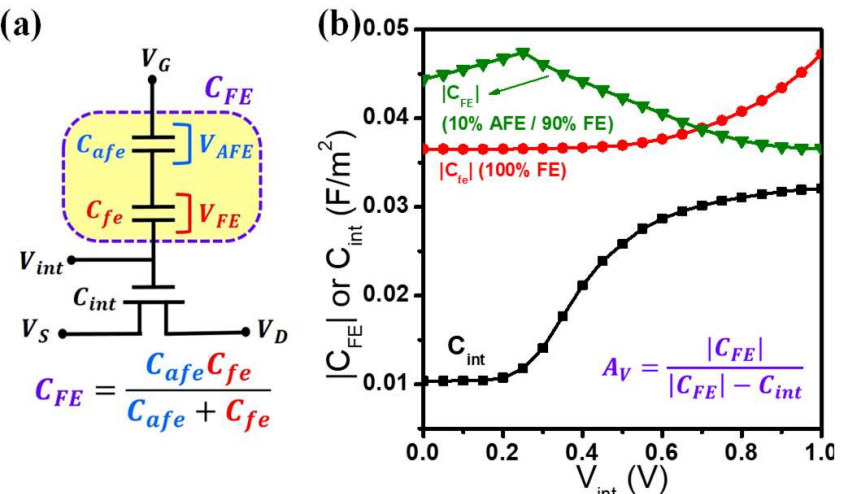

Figure 5. (a) Equivalent capacitance network of the AFE/FE stack NC-FinFET. The $\mathrm{C}_{\mathrm{afe}}, \mathrm{C}_{\mathrm{fe}}$ and $\mathrm{C}_{\mathrm{int}}$ are the anti-ferroelectric capacitance, ferroelectric capacitance and the internal capacitance, respectively. (b) Capacitance matching comparison at source end shows that the AFE/FE stack improves the high $\mathrm{A}_{\mathrm{V}}$ region toward high $\mathrm{V}_{\mathrm{GS}}$.

single-layer NC-FinFETs simulation results are achieved by our model. Here $3.6 \mathrm{~nm}$ ferroelectric thickness and $1 \mathrm{~nm}$ interfacial-layer thickness are used for hysteresis-free operation. It can be seen that, after the action of negative capacitance, the subthreshold swing can be improved from 61 to $48 \mathrm{mV} /$ decade for single-layer NCFET and to $49 \mathrm{mV} /$ decade for AFE/FE stack NCFET, respectively. Besides improved SS and OFF-state current (I $\mathrm{I}_{\text {OFF }}$ ), AFE/FE stack (with 10\% AFE) NC-FinFETs can achieve higher ON-state current $\left(\mathrm{I}_{\mathrm{ON}}\right)$ compared to that of the single-layer NCFET and non-NC FET. In this paper, 10\% AFE means the thickness of $\mathrm{AFE}\left(\mathrm{T}_{\mathrm{AFE}}\right)$ is $10 \%$ of the total ferroelectric thickness $\left(\mathrm{T}_{\mathrm{FE}}+\mathrm{T}_{\mathrm{AFE}}\right)$. The anti-ferroelectric parameters used in this paper were obtained based on the saturation polarization $\left(\mathrm{P}_{\mathrm{s}}=20 \mu \mathrm{C} / \mathrm{cm}^{2}\right)$ and dielectric constant $\left(\varepsilon_{\mathrm{AFE}}=25 \varepsilon_{0}\right)$ in [14]. The ferroelectric parameters of $\mathrm{HfZrO}$ used in this paper are: $\alpha_{\mathrm{FE}}=-3.8 \times 10^{9} \mathrm{~m} / \mathrm{F}, \beta_{\mathrm{FE}}=3.37 \times 10^{11}$ $\mathrm{m}^{5} / \mathrm{F} / \mathrm{C}^{2}$, and $\gamma_{\mathrm{FE}}=0 \mathrm{~m}^{9} / \mathrm{F} / \mathrm{C}^{4}$. These values of $\alpha_{\mathrm{FE}}, \beta_{\mathrm{FE}}$, and $\gamma_{\mathrm{FE}}$ were obtained based on the remnant polarization $\left(\mathrm{P}_{\mathrm{r}}\right)$ and coercive field $\left(\mathrm{E}_{\mathrm{c}}\right)$ data of $\mathrm{HfZrO}$ in [23].

\section{S-CURVE AND CAPACITANCE MATCHING OF AFE/FE STACK NC-FINFET}

By using the MFIS-type AFE/FE stack NC-FinFET model calculation, we investigate the impact of anti-ferroelectric on S-curve and capacitance matching for AFE/FE stack NC-FinFET in this section.

Fig. 4(a) and (b) show the P-V curves for $100 \% \mathrm{FE}$ and $100 \%$ AFE films, respectively. It can be seen that the negative capacitance region (negative slope of $\mathrm{P}-\mathrm{V}$ curve) for $100 \% \mathrm{FE}$ [Fig. 4(a)] starts from zero voltage and the magnitude of $\mathrm{C}_{\mathrm{FE}}$ decreases (i.e., $A_{V}$ increases) with increasing $\alpha_{\mathrm{FE}}$. Different from the ferroelectric, the anti-ferroelectric exhibits a double negative-capacitance region [as shown in Fig. 4(b)] occurring at higher voltage. Moreover, the magnitude of $\mathrm{C}_{\mathrm{AFE}}$ decreases with increasing $\alpha_{n}$. Therefore, if the AFE and FE are stacked in series, it is possible that by carefully choosing the parameters $\alpha_{\mathrm{FE}}$ and $\alpha_{\mathrm{n}}$, the AFE/FE stack can exhibit negative capacitance over a larger voltage range than that of the single-layer FE.

In this work, $\alpha_{\mathrm{FE}}=-3.8 \mathrm{e} 9 \mathrm{~m} / \mathrm{F}$ and $\alpha_{\mathrm{n}}=-1.2 \mathrm{e} 10 \mathrm{~m} / \mathrm{F}$ are used for the AFE/FE stack NC-FinFET to achieve larger $A_{V}$ with hysteresis-free operation. Fig. 4(c) shows the P-V curve 

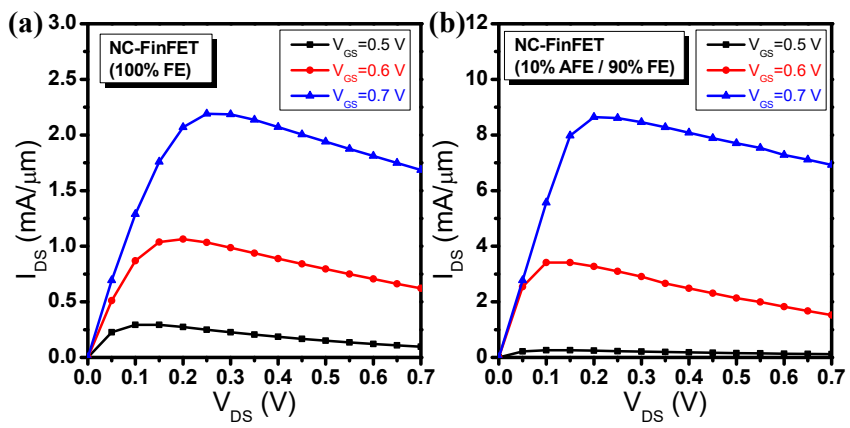

Figure 6. The output characteristics for (a) single-layer and (b) AFE/FE stack NC-FinFETs. The AFE/FE stack NC-FinFET exhibits higher $\mathrm{I}_{\mathrm{DS}}$ with increasing $\mathrm{V}_{\mathrm{GS}}$.

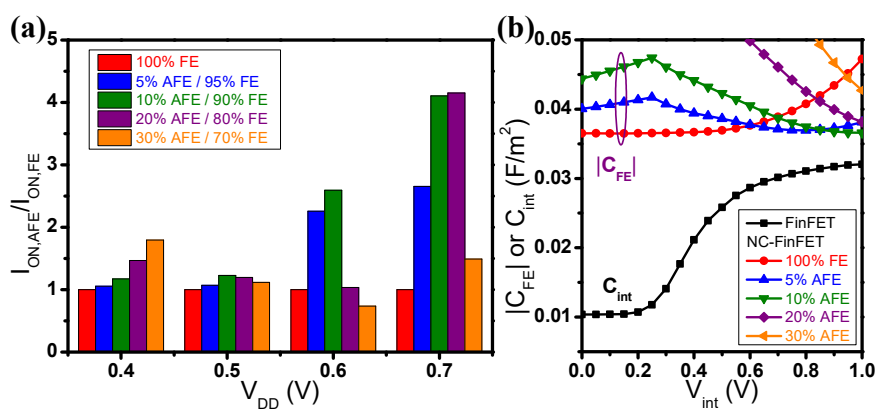

(c) 10 Open symbol: trajectory from $\mathrm{V}_{\mathrm{GS}}=0$ to $0.7 \mathrm{~V}$

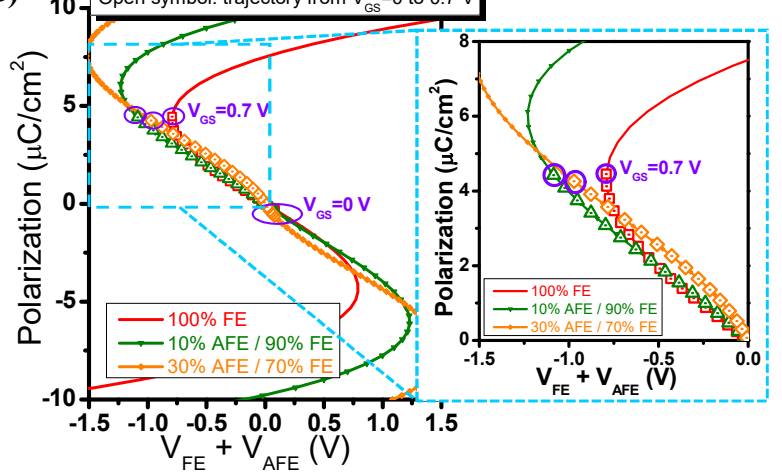

Figure 7. (a) Intrinsic $\mathrm{I}_{\mathrm{ON}}$ ratio for $\mathrm{AFE} / \mathrm{FE}$ stack to single-layer NC-FinFET with various $\mathrm{V}_{\mathrm{DD}}$ and $\%$ of $\mathrm{AFE}$. (b) Capacitance matching at source end and (c) S-curve comparison for AFE/FE stack NC-FinFET with various \% of AFE.

comparison for the $100 \% \mathrm{FE}$ and the $\mathrm{AFE} / \mathrm{FE}$ stack cases. It can be seen that the trajectory from $\mathrm{V}_{\mathrm{GS}}=0$ to $0.7 \mathrm{~V}$ are all in the negative capacitance region for the AFE/FE gate-stack. However, the operating point at $\mathrm{V}_{\mathrm{GS}}=0.7 \mathrm{~V}$ for the single-layer $\mathrm{FE}$ case moves away from the $\mathrm{NC}$ region, resulting in degraded Av. In other words, the AFE/FE gate-stack can be utilized toenable large gains in strong inversion for NC-FinFETs.

Fig. 5(a) shows the equivalent circuit of the AFE/FE stack NC-FinFET. Although the single-layer NC-FinFET leads to internal-voltage amplification in the subthreshold region, the increasing $\left|\mathrm{C}_{\mathrm{fe}}\right|$ at high $\mathrm{V}_{\mathrm{GS}}$ degrades the voltage-gain $\left(\mathrm{A}_{\mathrm{V}}=\right.$ $\left.\mid \mathrm{C}_{\mathrm{FE}} /\left(\left|\mathrm{C}_{\mathrm{FE}}\right|-\mathrm{C}_{\mathrm{int}}\right)\right)$ in strong inversion. Note that a higher $\mathrm{A}_{\mathrm{V}}$ can be obtained if $\left|\mathrm{C}_{\mathrm{FE}}\right|$ is closer to $\mathrm{C}_{\mathrm{int}}$. Therefore, by adding some anti-ferroelectric, the effective ferroelectric capacitance $\left(\mathrm{C}_{\mathrm{FE}}\right)$ is the series combination of the two capacitors (i.e. $\mathrm{C}_{\mathrm{FE}}=\mathrm{C}_{\mathrm{fe}} \| \mathrm{C}_{\mathrm{afe}}$ ) and can be engineered to improve $\mathrm{ON}$-state current $\left(\mathrm{I}_{\mathrm{ON}}\right)$. With first choosing the $\mathrm{C}_{\mathrm{fe}}$ to optimize the subthreshold swing, the AFE can be chosen to extend the negative $\mathrm{C}_{\mathrm{FE}}$ into the
Table 1. IRDS for 2020 to 2031 technology node [20].

\begin{tabular}{|c|c|c|c|c|c|}
\hline & \multicolumn{2}{|c|}{ FinFET } & \multicolumn{3}{|c|}{ Gate-all-around (GAA) } \\
\hline Year (node) & $2020(5)$ & $2022(3)$ & 2025 & $2028(1.5)$ & 2031 (1) \\
\hline Mainstream device & FinFET & FinFET & LGAA & LGAA & LGAA \\
\hline $\mathrm{L}_{\mathrm{g}}(\mathrm{nm})$ & 18 & 16 & 14 & 12 & 12 \\
\hline Fin width, $W_{\text {fin }}(n m)$ & 7 & 6 & & & \\
\hline Fin height, $H_{\text {fin }}(n m)$ & 50 & 60 & & & \\
\hline GAA width, $\mathrm{W}_{\mathrm{GAA}}(\mathrm{nm})$ & & & 25 & 20 & 15 \\
\hline GAA thickness, $\mathbf{T}_{\mathrm{GAA}}(\mathrm{nm})$ & & & 7 & 6 & 5 \\
\hline \# of stacked GAA & & & 3 & 3 & 4 \\
\hline Effective width, $W_{\text {eff }}(\mathrm{nm})$ & 107 & 126 & 192 & 156 & 160 \\
\hline $\mathrm{V}_{\mathrm{DD}}(\mathrm{V})$ & 0.7 & 0.7 & 0.65 & 0.65 & 0.6 \\
\hline SS (mV/dec.) & 78 & 82 & 80 & 75 & 75 \\
\hline $\begin{array}{l}\text { ntrinsic } I_{O N}(\mu \mathrm{A} / \mu \mathrm{m}) \\
\left.\text { w/ } \mathbf{I}_{\mathrm{OFF}}=1 \mathrm{e}-8\right)\end{array}$ & 1733 & 1950 & 1670 & 1975 & 1696 \\
\hline
\end{tabular}
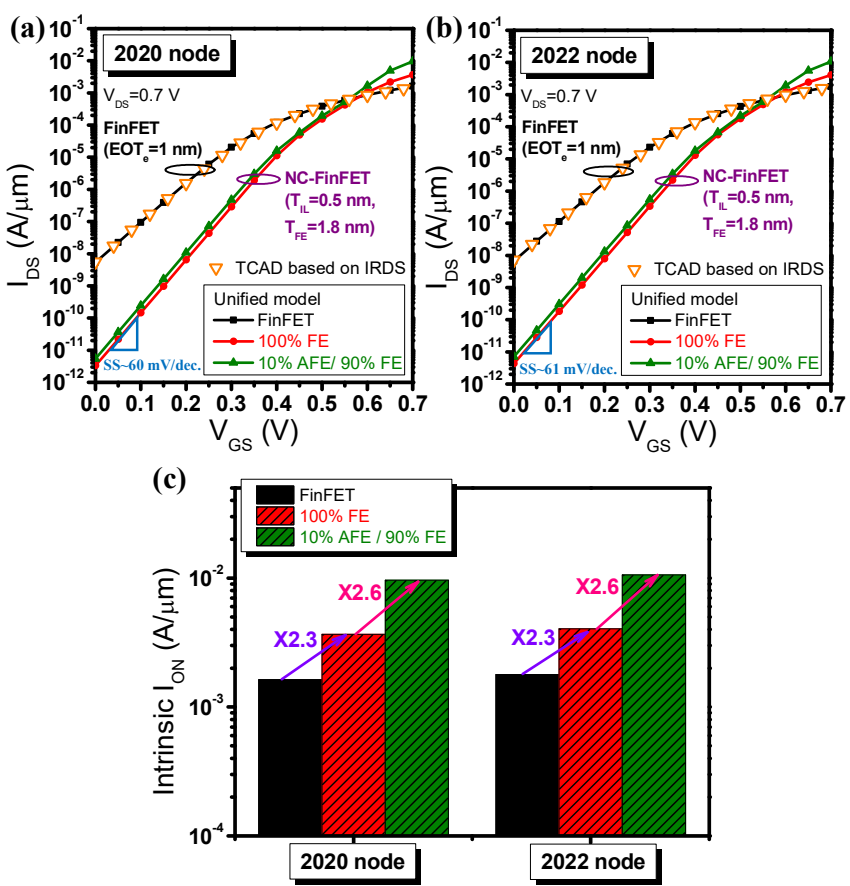

Figure 8. $\mathrm{I}_{\mathrm{DS}}-\mathrm{V}_{\mathrm{GS}}$ characteristics comparison for NC-FinFET and FinFET in (a) 2020 and (b) 2022 node. (c) Comparison of intrinsic I $\mathrm{I}_{\mathrm{N}}$ for NC-FinFET and 2020/2022 FinFET.

strong inversion region to obtain the overall good capacitance matching. Fig. 5(b) shows the comparison of capacitance matching at the source end for different gate-stacks. It can be seen that the NC-FinFET with AFE/FE stack achieves a relatively flat negative capacitance at higher $\mathrm{V}_{\mathrm{GS}}$, achieving high- $A_{V}$ in that region.

Fig. 6 shows the output characteristics of the single-layer and AFE/FE stack NC-FinFETs with various $\mathrm{V}_{\mathrm{GS}}$. It can be seen that the AFE/FE stack NC-FinFET can achieve larger IDS than that of the single-layer due to the better capacitance matching and higher $A_{V}$ in strong inversion. In reality, source-drain series resistance will make such high $\mathrm{I}_{\mathrm{DS}}$ impractical. The likely strategy is to use lower $\mathrm{V}_{\mathrm{DD}}$ to achieve much lower power consumption.

Fig 7(a) shows the ratio of intrinsic $\mathrm{I}_{\mathrm{ON}}$ for AFE/FE stack to single-layer NC-FinFETs ( $\mathrm{I}_{\mathrm{ON}, \mathrm{AFE}} / \mathrm{I}_{\mathrm{ON}, \mathrm{FE}}$ ) with varies $\mathrm{V}_{\mathrm{DD}}$ and $\%$ of AFE. It can be seen that the optimum \% (higher $\left.\mathrm{I}_{\mathrm{ON}, \mathrm{AFE}} / \mathrm{I}_{\mathrm{ON}, \mathrm{FE}}\right)$ of AFE is about $10 \%$ for $\mathrm{V}_{\mathrm{DD}}>0.5 \mathrm{~V}$. As AFE 


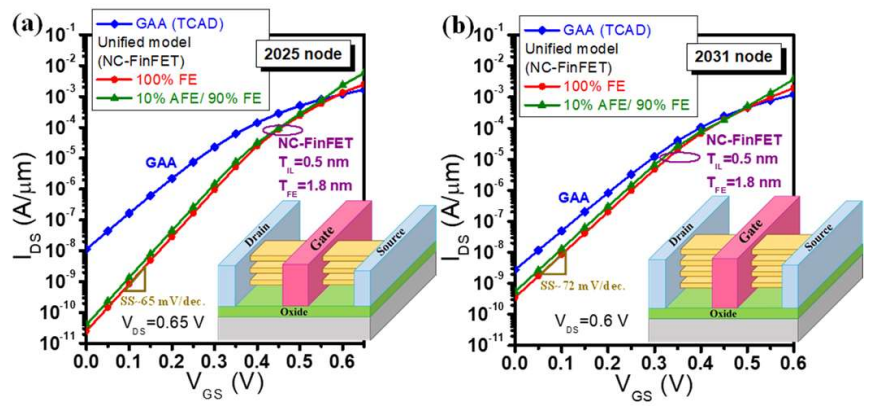

Figure 9. $\mathrm{I}_{\mathrm{DS}}-\mathrm{V}_{\mathrm{GS}}$ characteristics comparison for NC-FinFET and GAA in (a) 2025 and (b) 2031 node. Inset: schematic of stacked GAA for each node.

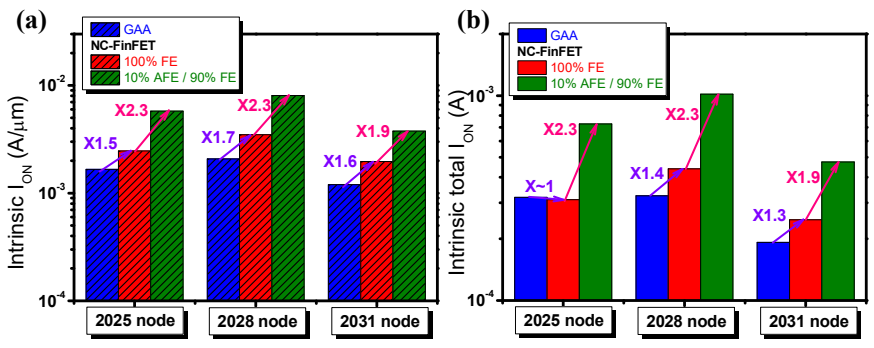

Figure 10. Comparison of (a) intrinsic $\mathrm{I}_{\mathrm{ON}}$ and (b) total intrinsic $\mathrm{I}_{\mathrm{ON}}$ for NCFinFET and 2025 2031 stacked GAA. Here the fin-height for NC-FinFET is $60 \mathrm{~nm}$ (spec. of IRDS in 2022 node).

increases beyond the optimum \%, I IN,AFE degrades rapidly. This is because the capacitance matching is dominated by AFE in strong inversion. Fig. 7(c) shows that, the slope of S-curve at low-voltage increase with $\%$ of AFE, which degrades the $A_{V}$ in the threshold region and moves the high- $A_{V}$ region to higher $\mathrm{V}_{\mathrm{GS}}$ [Fig. 7(b)]. Furthermore, for low $\mathrm{V}_{\mathrm{DD}}(<0.5 \mathrm{~V})$ condition, the capacitance matching is dominated by FE due to the $A_{V}$ degradation of AFE at low voltage.

\section{PROJECTION BASED ON IRDS}

The projection of IRDS for future FinFET and GAA are shown in [20] (Table. I). Using the parameters in the table, we simulate the potential performance improvement that $\mathrm{FE}$ and $10 \%$-AFE/FE stack can theoretically achieve if AFE and FE materials assumed in our study are available. Our model is calibrated with each IRDS high performance 2020 2031 node through TCAD simulation before the FE or AFE-FE stack is introduced. Besides, in order to meet the tight pitch requirement for future advanced nodes, $1.8 \mathrm{~nm} \mathrm{~T}_{\mathrm{FE}}$ and $0.5 \mathrm{~nm} \mathrm{~T}_{\mathrm{IL}}$ are used in this section.

Fig 8(a) and (b) show the $\mathrm{I}_{\mathrm{DS}}-\mathrm{V}_{\mathrm{GS}}$ characteristics FinFET and NC-FinFETs (single-layer and AFE/FE) for the 2020 and 2022 nodes, respectively. NC-FinFETs can theoretically achieve steeper SS and reduce OFF-state current (I $\left.\mathrm{I}_{\mathrm{OFF}}\right)$, which can significantly reduce the static-power. Besides, AFE/FE stack NC-FinFETs have higher $\mathrm{I}_{\mathrm{ON}}\left(\mathrm{I}_{\mathrm{DS}}\right.$ at $\left.\mathrm{V}_{\mathrm{GS}}=\mathrm{V}_{\mathrm{DD}}\right)$ than the single-layer counterparts. The comparison of intrinsic Ion for NC-FinFETs and 2020/2022 FinFET are shown in Fig. 8(c). It can be seen that the intrinsic ION of NC-FinFET with AFE/FE stack can be further improved $\sim 2.6 \mathrm{X}$ than the single-layer, which means that the AFE/FE stack may reduce the pressure in fin-height up-scaling.
The IRDS Table. I shows that the mainstream device would change from FinFET to gate-all-around (GAA) due to electrostatic integrity requirement. The $\mathrm{I}_{\mathrm{DS}}-\mathrm{V}_{\mathrm{GS}}$ characteristics comparison in Fig. 9 shows that the NC-FinFET can exhibit superior subthreshold IV than GAA counterparts for 2025 and 2031 nodes. Furthermore, Fig. 10(a) shows the comparison of intrinsic ION for NC-FinFET and 2025 2031 GAA. It can be seen that, while the intrinsic ION for the single-layer NC-FinFETs is potentially larger $(\sim 1.5 \mathrm{X})$ than that of GAA, the intrinsic $\mathrm{I}_{\mathrm{ON}}$ for the NC-FinFETs with AFE/FE stack can be further improved to $\sim 2 \mathrm{X}$.

Fig. 10(b) shows the comparison of total intrinsic ION for 2025 2031 GAA (inset in Fig. 9) and NC-FinFET. Here the fin-height for NC-FinFET is $60 \mathrm{~nm}$ (spec. of IRDS in 2022 node). It shows that, due to the technology process limitation of fin-height for NC-FinFET, the total intrinsic $I_{O N}$ for single-layer is comparable to the stacked GAA in 2025 node. By adding some AFE, the total intrinsic $\mathrm{I}_{\mathrm{ON}}$ can be further improved $\sim 2.3 \mathrm{X}$ for the AFE/FE stack NC-FinFET. Besides, the total intrinsic $\mathrm{I}_{\mathrm{ON}}$ of GAA slightly decreases from 2028 to 2031 node. The AFE/FE stack NC-FinFET can help to improve ION. In other words, NC-FinFET with AFE/FE stack has the

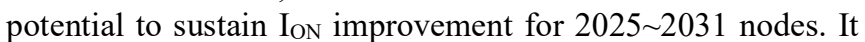
should be noted that this theoretical study assumes the availability of materials with certain AFE and FE properties, and the $\mathrm{AFE} / \mathrm{FE}$ stack is purely capacitive. In reality, implementing such a thin AFE/FE stack without resistive components is very challenging, and demands more research in the future.

\section{CONCLUSION}

In this work, we report that the AFE/FE gate-stack can be utilized to engineer the S-curve for boosting the $\mathrm{I}_{\mathrm{ON}}$ of NC-FinFET. By using a short-channel BSIM-CMG compatible AFE/FE stack NC-FinFET model, the capacitance matching and ON-state performance for AFE/FE stack NC-FinFETs are investigated. Our study indicates that, the AFE/FE gate-stack can be used to improve the capacitance matching in strong inversion at higher gate-bias. Therefore, impressively higher ON-state current (compared to single-layer) can be achieved. In reality, source-drain series resistance will make such high $\mathrm{I}_{\mathrm{DS}}$ impractical. The more likely strategy is to use lower $V_{D D}$ to achieve much lower power consumption (and reduced vertical fields). While the transient NC effect [25][26] also needs to be carefully investigated, this study suggests significant long term benefits to $V_{D D}$ scaling if materials with certain AFE and FE properties are developed and introduced in IC manufacturing in the future.

\section{ACKNOWLEDGMENT}

The authors would like to thank anonymous referees for critical reading of the manuscript and valuable feedback.

\section{REFERENCES}

[1] S. Salahuddin and S. Datta, "Use of negative capacitance to provide voltage amplification for low power nanoscale devices," Nano Letters, pp 405-410, Feb. 2008.

[2] Z. Krivokapic, U. Rana, R. Galatage, A. Razavieh, A. Aziz, J. Liu, J. Shi, 
H. J. Kim, R. Sporer, C. Serrao, A. Busquet, P. Polakowski, J. Müller, W. Kleemeier, A. Jacob, D. Brown, A. Knorr, R. Carter, and S. Banna, "14nm ferroelectric FinFET technology with steep subthreshold slope for ultra low power applications," IEDM Tech. Dig., Dec. 2017, pp. 15.1.1-4.

[3] K.-S. Li, P.-G. Chen, T.-Y. Lai, C.-H. Lin, C.-C. Cheng, C.-C. Chen, Y.- J. Wei, Y.-F. Hou, M.-H. Liao, M.-H. Lee, M.-C. Chen, J.-M. Sheih, W.- K. Yeh, F.-L. Yang, S. Salahuddin and C. Hu, "Sub-60 mV-swing negative-capacitance FinFET without hysteresis," IEDM Tech. Dig., Dec. 2015, pp. 22.6.1-4.

[4] E. Ko, J. W. Lee and C. Shin, "Negative capacitance FinFET with sub-20-mV SS and minimal hysteresis of $0.48 \mathrm{~V}$," IEEE EDL, Vol. 38, No. 4 Apr. 2017.

[5] P. Su and W. X. You, "Electrostatic integrity in negative-capacitance FETs - A subthreshold modeling approach," IEDM Tech. Dig., Dec. 2019, pp. 7.3.1-7.3.4.

[6] H. Agarwal, P. Kushwaha, Y.-K. Lin, M.-Y. Kao, Y.-H. Liao, A. Dasgupta, S. Salahuddin, and Chenming Hu, "Proposal for Capacitance matching in negative capacitance field-effect transistors," in IEEE Electron Device Letters, vol. 40, no. 3, pp. 463-466, March 2019.

[7] S.-E. Huang, C.-L. Yu, and Pin Su, "Investigation of fin-width sensitivity of threshold voltage for InGaAs and Si negative-capacitance FinFETs considering quantum-confinement effect," IEEE Trans. Electron Devices, vol. 66, no. 6, pp. 2538-2543 , Jun. 2019

[8] W. X. You, P. Su and C. Hu, "Evaluation of NC-FinFET based subsystem-level logic circuits," in IEEE Transactions on Electron Devices, vol. 66, no. 4, pp. 2004-2009, April 2019.

[9] J. P. Duarte et al., "Compact models of negative-capacitance FinFETs: Lumped and distributed charge models," in IEDM Tech. Dig., Dec. 2016 , pp. 30.5.1-30.5.4. doi: 10.1109/IEDM.2016.7838514.

[10] H. Ota, T. Ikegami, J. Hattori, K. Fukuda, S. Migita, and A. Toriumi, "Fully coupled 3-D device simulation of negative capacitance FinFETs for sub $10 \mathrm{~nm}$ integration," in IEDM Tech. Dig., Dec. 2016, pp. 318-321.

[11] W.-X. You, C.-P. Tsai, and P. Su, "Short-channel effects in 2D negativecapacitance field-effect transistors," IEEE Trans. Electron Devices, vol. 65, no. 4, pp. 1604-1610, Apr. 2018.

[12] D. Kwon, K. Chatterjee, A. J. Tan, A. K. Yadav, H. Zhou, A. B. Sachid, R. Reis, C. Hu, and S. Salahuddin, "Improved subthreshold swing and short channel effect in FDSOI n-channel negative capacitance field effect transistors," IEEE Electron Device Letters, vol. 39, no. 2, pp. 300-303, Feb. 2018.

[13] J. Müller et al., "Ferroelectricity in simple binary $\mathrm{ZrO}_{2}$ and $\mathrm{HfO}_{2}$," in Nano Lett., vol. 12, no. 8, pp. 4318-4323, 2012, doi: 10.1021/nl302049k

[14] X. Lyu, M. Si, X. Sun, M. A. Capano, H. Wang and P. D. Ye, "Ferroelectric and anti-ferroelectric hafnium zirconium oxide: scaling limit, switching speed and record high polarization density," 2019 Symposium on VLSI Technology, Kyoto, Japan, 2019, pp. T44-T45.

[15] K. Karda, A. Jain, C. Mouli, and M. A. Alam, "An anti-ferroelectric gated Landau transistor to achieve sub- $60 \mathrm{mV} / \mathrm{dec}$ switching at low voltage and high speed," Applied Physics Letters, 106, 163501, Apr., 2015.

[16] Y.-T Tang, C.-J. Su, Y.-S. Wang, K.-H. Kao, T.-L. Wu, P.-J. Sung, F.-J. Hou, C.-J. Wang, M.-S. Yeh, Y.-J. Lee, W.-F. Wu, G.-W. Huang, J.-M Shieh, W.-K. Yeh, and Y.-H. Wang, "A comprehensive study of polymorphic phase distribution of ferroelectric-dielectrics and interfacial layer effects on negative capacitance FETs for sub-5 nm node," 2018 IEEE Symposium on VLSI Technology, Honolulu, 2018, pp. 45-46.

[17] M. H. Lee, K.-T. Chen, C.-Y. Liao, G.-Y. Siang, C. Lo, H.-Y. Chen, Y.-J. Tseng, C.-Y. Chueh, C. Chang, Y.-Y. Lin, Y.-J. Yang, F.-C. Hsieh, S. T. Chang, M.-H. Liao, K.-S. Li, and C. W. Liu, "Bi-directional sub- $60 \mathrm{mV} / \mathrm{dec}$, hysteresis-free, reducing onset voltage and high speed response of ferroelectric-antiferroelectric $\mathrm{Hf}_{0.25} \mathrm{Zr}_{0.75} \mathrm{O}_{2}$ negative capacitance FETs," 2019 IEEE International Electron Devices Meeting (IEDM), San Francisco, CA, USA, 2019, pp. 23.6.1-23.6.4, doi: 10.1109/IEDM19573.2019.8993581.

[18] (2016). BSIM-CMG Technical Manual. [Online]. Available: http:// bsim.berkeley.edu/models/bsimcmg/.

[19] C.-H. Jan et al., "A $14 \mathrm{~nm}$ SoC platform technology featuring 2nd generation tri-gate transistors, $70 \mathrm{~nm}$ gate pitch, $52 \mathrm{~nm}$ metal pitch, and 0.0499 um2 SRAM cells, optimized for low power, high performance and high density SoC products," in VLSI Symp. Tech. Dig., Jun. 2015, pp. T12-T13. doi: 10.1109/VLSIC.2015.7231380.

[20] IRDS (https://irds.ieee.org/).

[21] C. Kittel, "Theory of antiferroelectric crystals," Phys. Rev. 82, 729, Jun. 1951.

[22] L. D. Landau and I. M. Khalatnikov, "On the anomalous absorption of sound near a second order phase transition point," Dokl. Akad. Nauk SSSR, vol. 96, pp. 469-472, 1954.

[23] M. Kobayashi and T. Hiramoto, "Device design guideline for steep slope ferroelectric FET using negative capacitance in sub- $0.2 \mathrm{~V}$ operation: Operation speed, material requirement and energy efficiency," in $V L S I$ Symp. Tech. Dig., Jun. 2015, pp. T212-T213.

[24] Sentaurus Process User Guide, Version O-2018.06, Synopsys, Mountain View, CA, USA, Sep. 2018.

[25] M. Hoffmann et al., "Demonstration of high-speed hysteresis-free negative capacitance in ferroelectric $\mathrm{Hf}_{0.5} \mathrm{Zr}_{0.5} \mathrm{O}_{2}$," IEDM Tech. Dig., Dec. 2018, pp. 31.6.1-31.6.4

[26] C. Gastaldi, A. Saeidi, M. Cavalieri, I. Stolichnov, P. Muralt and A. M. Ionescu, "Transient Negative Capacitance of silicon-doped $\mathrm{HfO} 2$ in MFMIS and MFIS structures: experimental insights for hysteresis-free steep slope NC FETs," 2019 IEEE International Electron Devices Meeting (IEDM), San Francisco, CA, USA, 2019, pp. 23.5.1-23.5.4, doi: 10.1109/IEDM19573.2019.8993523. 